\title{
Quand le terrain est un monastère bouddhique tibétain
}

When the field is a Tibetan Buddhist monastery

\section{Mireille Helffer}

\section{(2) OpenEdition}

\section{Journals}

Édition électronique

URL : http://journals.openedition.org/ethnomusicologie/1190

ISSN : 2235-7688

Éditeur

ADEM - Ateliers d'ethnomusicologie

Édition imprimée

Date de publication : 31 décembre 1995

Pagination : 69-84

ISBN : 2-8257-0537-3

ISSN : $1662-372 X$

\section{Référence électronique}

Mireille Helffer, "Quand le terrain est un monastère bouddhique tibétain », Cahiers d'ethnomusicologie [En ligne], 8| 1995, mis en ligne le 04 janvier 2012, consulté le 30 avril 2019. URL : http:// journals.openedition.org/ethnomusicologie/1190

Ce document a été généré automatiquement le 30 avril 2019.

Tous droits réservés 


\title{
Quand le terrain est un monastère bouddhique tibétain
}

When the field is a Tibetan Buddhist monastery

\author{
Mireille Helffer
}

1 Lorsqu'au début des années 1970, j'ai décidé d'entreprendre une recherche sur les notations utilisées dans la tradition musicale du bouddhisme tibétain, la documentation existante était extrêmement limitée et surtout aucun lien n'avait encore été établi entre documents écrits (conservés dans les bibliothèques d'Occident) et documents sonores qui, grâce aux nouvelles techniques d'enregistrement, commençaient à se répandre sur le marché occidental et révélaient la richesse et l'originalité de la musique rituelle tibétaine ${ }^{1}$

2 Après avoir procédé à un inventaire des manuscrits existant dans les grandes bibliothèques occidentales et après une brève enquête à l'institut tibétain de Rikon/ Zurich (1972) où j'avais fini par découvrir un moine tibétain capable de lire une de ces mystérieuses notations et l'avais enregistré, je présentai le résultat de mes investigations à un public de tibétologues lors du xxIX congrès des Orientalistes (1973) ${ }^{2}$. Devant les réactions favorables et les encouragements prodigués par mes collègues tibétologues, une première mission exploratoire en Inde parut possible. Cette mission marqua le début d'une longue période de recherches durant laquelle le terrain a tenu une place capitale, même si le temps passé en « laboratoire » à déchiffrer, écouter, analyser les documents recueillis était porportionnellement beaucoup plus considérable.

3 C'est à une réflexion sur l'évolution de ma pratique de terrain au cours des vingt dernières années que je tenterai de me livrer ici, dans l'espoir qu'elle pourra être utile à ceux ou celles qui seraient amenés à reprendre le flambeau.

\section{Les conditions de la recherche}

$4 \quad$ Au Tibet proprement dit, qu'il s'agisse de ce qui est devenu Région Autonome du Tibet ou des provinces orientales du Khams et de l'Amdo, la situation politique était telle qu'on 
pouvait craindre la disparition totale des monastères et l'extermination des moines dépositaires des traditions... d'ailleurs aucun chercheur occidental n'aurait pu obtenir des autorités chinoises les permis nécessaires.

La seule solution envisageable était donc de se tourner vers les Tibétains de l'exil qui, fuyant l'invasion chinoise, avaient trouvé refuge dans les régions frontalières (royaumes du Népal, du Sikkim ou du Bhoutan) et en divers points de l'Inde (Himachal Pradesh, Orissa, Karnataka), là où le gouvernement indien leur avait octroyé des terres. Mais ce n'était pas là entreprise facile, étant donné la dispersion des camps tibétains et l'absence de structure scientifique d'accueil aussi bien du côté tibétain, que du côté indien ou népalais. De 1973 à aujourd'hui, j'ai effectué ainsi une dizaine de missions en Inde et au Népal, visitant de nombreux monastères où, selon les possibilités qui m'étaient offertes et l'accueil qui m'était fait, je passais quelques heures, quelques jours ou plusieurs semaines.

\section{Le choix des terrains}

6 A la fin de 1973, j'entrepris donc une première mission exploratoire en Inde ; il s'agissait alors dans mon esprit de procéder à un repérage géographique des communautés tibétaines se réclamant des différentes écoles du bouddhisme tibétain qui pouvaient avoir conservé des notations musicales propres à leur tradition et de savoir si, simultanément, elles comptaient en leur sein des «maîtres de chant » (dbu-mdzad) compétents, capables de traduire au plan sonore le graphisme assez sommaire des neumes tibétains et disposés à les faire enregistrer.

7 Je disposais de quelques informations recueillies par mon collègue et ami tibétologue Yoshiro Imaeda qui, en 1972, avait non seulement photographié à mon intention un manuel de chants qu'avait reconstitué de mémoire un ancien dbu-mdzad du monastère dge-lugs-pa de Rnam-rgyal grwa-tshang à Dharamsala, mais avait également procédé à quelques enregistrements avec ce même dbu-mdzad. Mais c'est Gene Smith, animateur infatigable d'un programme de publication des ouvrages tibétains initié par la Library of Congress à New- Delhi, qui m'aida, dès mon arrivée, à établir un plan de bataille qui me permit de rencontrer des représentants autorisés des quatre principales écoles du bouddhisme tibétain - dge-lugs-pa, sa-skya-pa, bka'-brgyud-pa, rnying-ma-pa - et s'employa à me faciliter la tâche par ses recommandations efficaces.

C'est ainsi que j'ai visité des communautés dge-lugs-pa à Dharamsala et Dalhousie, le monastère de Khams-sprul Rinpoche à Tashijong, les Centres sa-skya-pa et rnying-ma-pa de Dehra Dun, avant d'atteindre le monastère de Thugs-sras Rinpoche à Darjeeling; dans chacun de ces lieux, il me fallait tenter d'expliciter une démarche qui avait tout pour surprendre des religieux préoccupés avant tout de rétablir des traditions liturgiques dans des conditions matérielles souvent précaires et avec un nombre encore très limité de moines! Néanmoins, au bout de trois mois de quête, le bilan s'avéra positif, tant au point de vue des traditions de notations identifiées qu'à celui des enregistrements effectués (Paris, Musée de l'Homme : BM 90-10-1 à 10) : tous les monastères contactés avaient ou avaient eu des manuels de notations musicales et cherchaient à en rétablir l'usage en exil. Si, au plan culturel, je pouvais être satisfaite, je ne pouvais pas m'empêcher d'être déçue par le côté dépouillé d'un mode de psalmodie s'attachant beaucoup plus à différencier des timbres qu'à marquer une recherche mélodique. 
$9 \mathrm{Au}$ retour, commença alors un austère travail de décryptage des manuels recueillis photographiés ou parfois recopiés - (la photocopie n'était pas encore généralisée) pour essayer d'en comprendre et comparer les conventions d'écriture et le vocabulaire, tout en m'appuyant sur les documents sonores qui les illustraient (Helffer, 1976).

10 Dans les années qui suivirent, de 1975 à 1985, les publications en fac-similé de recueils de notations musicales tibétaines se multiplièrent, portant à plusieurs milliers de pages la documentation écrite dont il y avait lieu de tenir compte (cf. la bibliographie publiée dans Helffer, 1994 :340-343). Simultanément, et dans une ignorance mutuelle quasi totale, d'autres chercheurs avaient abordé les mêmes sujets; les uns, comme Kaufmann ou Vandor, étaient musiciens de formation, d'autres, parmi lesquels Riccardo Canzio et Ter Ellingson, forts d'une longue expérience de terrain en Inde et au Népal, préparaient des Ph. D. qui devaient faire date. C'est avec intérêt et une certaine inquiétude que je guettais les publications des uns et des autres, craignant toujours d'être distanciée ou contredite!

11 En 1976, je me rendis au Ladakh, redevenu depuis peu accessible, dans le but précis de rencontrer les autorités du monastère 'Bri-gung bka'-brgyud-pa ${ }^{3}$ de Phyang dont un volumineux manuel de notations avait été publié l'année précédente (cf. Helffer, 1978). Je me trouvais là dans une situation différente puisque le monastère était une fondation ladakhie ancienne dont la situation apparaissait moins précaire que les fondations récentes des réfugiés tibétains et dont le fonctionnement, me semblait-il, pouvait être plus conforme à la tradition.

12 En 1979 et 1984, je poursuivis mes enquêtes en Inde, et étendis mes investigations au Népal où, à côté de monastères déjà anciens (comme ceux appartenant aux traditions karma bka'-brgyud-pa à Swayambhunath ou sa-skya-pa à Bodnath), commençaient à fleurir des monastères nouveaux créés par quelques-uns des grands maitres religieux qui avaient pris le chemin de l'exil à la suite du Dalaï lama et qui s'efforçaient de regrouper autour d'eux des tenants de la tradition à laquelle ils appartenaient et de recruter de jeunes moines auxquels transmettre ces traditions (Helffer, 1993a).

13 Enfin, depuis 1987, comme je l'ai expliqué dans deux publications (Helffer 1993b), j'ai choisi d'approfondir les traditions musicales rnying-ma-pa, un peu négligées jusque là, et me suis tournée vers deux importants monastères de la tradition rnying-ma-pa: le monastère de Dpal-yul à Bylakuppe en Inde, et le monastère de Zhe-chen à Bodnath au Népal, dans lesquels j’ai effectué des séjours de plus longue durée.

\section{Le choix de la période de l'année où effectuer les missions de terrain}

14 Il a d'abord été dicté par mes propres choix, effectués en tenant compte de deux impératifs principaux :

1. éviter des conditions climatiques défavorables, peu propices aux déplacements et aux enregistrements, c'est-à-dire les excès du plein hiver dans les régions himalayennes (Ladakh), comme l'humidité de la mousson... C'est la raison pour laquelle mes premières missions se sont effectuées à l'automne ou au début du printemps.

2. obéir aux exigences du calendrier universitaire : assurer l'enseignement auquel je m'étais engagée, et ne pas disparaitre au moment où les étudiants devaient rédiger et remettre leurs mémoires. 
15 J'ai mis un certain temps à me rendre compte qu'il ne s'agissait pas là d'une bonne politique. J'avais pensé tout d'abord qu'il fallait éviter de se rendre dans les monastères au moment des fêtes importantes qui risquaient de mobiliser les moines dans leurs fonctions liturgiques, mais je m'aperçus qu'il n'en était rien et que, tout au contraire, c'était à ces moments là que les moines étaient plus ou moins tenus d'être présents. Autrement, nombre d'entre eux s'absentaient, vaquaient à leurs affaires, étaient appelés à l'extérieur pour effectuer des rituels chez des particuliers, bref devenaient impossibles à joindre.

avait également le risque de tomber pendant la période de la « retraite estivale » (dbyar gnas) durant laquelle les moines ne sont pas supposés sortir du monastère, ce qui m'est arrivé en 1979 et m'a valu une longue attente à Darjeeling avant de pouvoir procéder à l'enquête prévue.

17 J'ai donc dû apprendre à tenir davantage compte non seulement du calendrier général des fêtes tibétaines, avec ses célébrations mensuelles, annuelles, exceptionnelles, mais aussi des calendriers propres à chacune des traditions considérées ; c'est ainsi qu'il m'est apparu que la période du Nouvel An tibétain (lo-sar) était particulièrement propice au déroulement des enquêtes de terrain en raison de l'importance et de la solennité des rituels qui se succédaient et donc de la place qui y était faite au chant et à l'emploi des instruments de musique.

Fig. 1 : Un religieux itinérant, en pèlerinage à Lhassa, récite des textes sacrés en s'accompagnant du tambour damaru et de la cloche dril-bu. Lhassa, 1991.

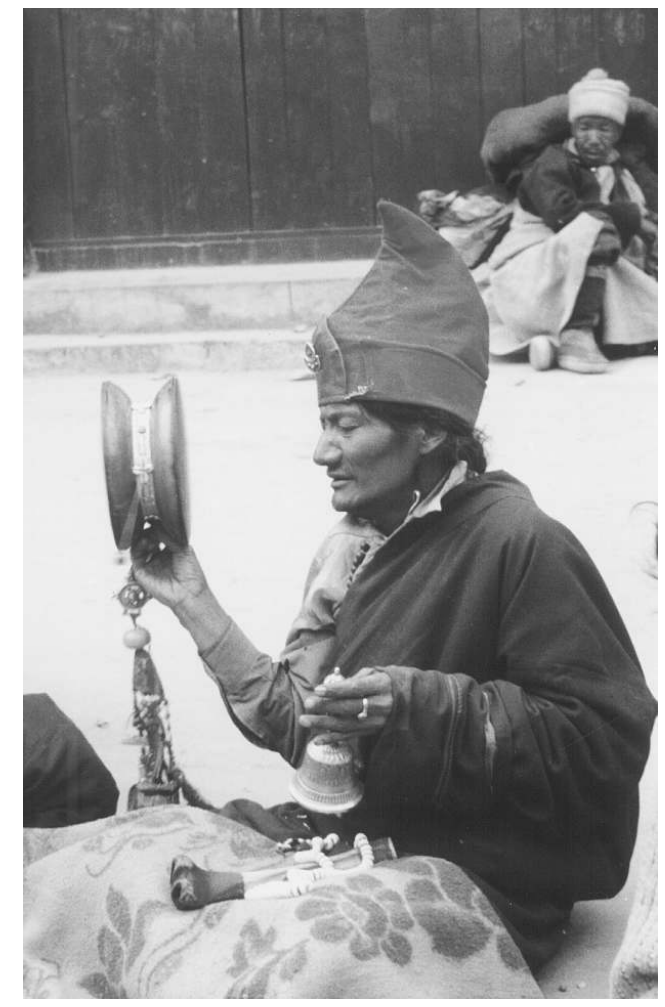

Photo : Mireille Helffer. 


\section{La collecte des données : poids des recommandations et reconnaissance des compétences}

18 Une fois sur les lieux et en espérant que la période choisie serait favorable, comment allais-je procéder?

Comme je l'ai dit plus haut, les données à recueillir en priorité m'apparaissaient être de deux ordres: l'ordre de l'écrit (les manuels de chant) et l'ordre de l'oral (les enregistrements qui pourraient servir de référence).

20 A qui m'adresser dans ces communautés monastiques naissantes ou déjà bien établies dont le rôle essentiel était de perpétuer une tradition et d'assurer aux fidèles les conditions d'une bonne renaissance, mais sûrement pas de répondre à la curiosité scientifique d'une étrangère, de surcroît non-bouddhiste?

21 La complexité de l'organisation monastique me paraissait extrême ; en effet, même si, à l'époque, je connaissais les distinctions entre "novices", "moines ordonnés", responsables de la discipline, maîtres de chant (dbu-mdzad), abbé (mkhan-po), maîtres du rituel (rdo-rje slob-dpon), réincarnés (sprul-sku), j'étais bien loin d'avoir compris comment fonctionnait le code subtil des hiérarchies respectives.

Il s'est avéré bien vite que le poids des recommandations dont je pouvais bénéficier était déterminant et j'en donnerai quelques exemples.

Lorsque, en 1973, je suis arrivée au Centre sa-skya-pa de Dehra Dun, j'avais au préalable rencontré à New-Delhi le pontife suprême de l'ordre sa-skya-pa et lui avais exposé le but de ma démarche ; par chance, il se trouvait sur place et c'est lui qui a intimé au « maître de chant", un moine âgé formé au Tibet, l'ordre d'enregistrer les dix-huit chants vocalisés pour le protecteur Mgon-po, dont j'avais découvert l'existence dans un texte de rituel. J'ai également obtenu de recopier les passages du manuel de chant correspondant à mes enregistrements, en attendant la parution annoncée d'une édition de ce manuel en Inde (parution qui eut effectivement lieu en 1977).

Pour contacter le « maître de chant » du monastère dge-lugs-pa de Rnam-rgyal-tshang à Dharamsala, je bénéficiais de deux avantages : une photo du manuel de chant (dbyangsyig) qu'il avait lui-même reconstitué et dont j'avais eu la copie grâce à mon collègue Yoshiro Imaeda, et une lettre de recommandation, accompagnée de cadeaux, que m'avait donnée Dvags-po Rinpoche qui enseignait alors le tibétain à Paris et qui connaissait personnellement ce maître de chant.

Je fus aussitôt en quelque sorte prise en main par le «maître de chant » qui s'occupa même de me trouver une chambre plus proche du monastère pour faciliter les contacts, et avec lequel je procédais à des enregistrements systématiques de quelques-uns des dbyangs les plus importants du manuel (Helffer, 1980).

Ailleurs, comme à Darjeeling, la recommandation venait de mon collègue Gene Smith qui avait lui-même séjourné à plusieurs reprises dans le monastère et était un disciple de Thugs-sras Rinpoche, la plus haute autorité de ce monastère ; une lettre de Gene Smith avait précédé mon arrivée et, en l'absence de Thugs-sras Rinpoche, c'est l'abbé du monastère qui prit toutes les dispositions nécessaires pour faciliter mon travail d'enregistrement. Je fis alors la connaissance d'un jeune moine qui, ayant fait ses études 
au collège jésuite de Darjeeling, parlait bien anglais et m'aida considérablement par la suite pour prendre des contacts utiles dans d'autres monastères.

Au Ladakh, j'étais porteuse d'une lettre adressée à un laïc influent qui fut fort difficile à joindre, mais qui se chargea d'organiser une séance d'enregistrement dans le monastère où je souhaitais travailler. Mais, dans les différents cas évoqués ci-dessus, je procédais aux enregistrements hors-situation, ils étaient effectués à ma demande, dans des conditions artificielles.

Même si c'était plus délicat à réaliser, il me fallait parvenir à effectuer des enregistrements en situation, pendant les rituels eux-mêmes. Ce n'est que plus tard, à partir de 1979 que j'y parvins, d'abord durant des rituels privés effectués au domicile de quelque particulier, puis durant des rituels réguliers dans un monastère dont je connaissais déjà le «maître de chant ». Enfin la présence du moine français Matthieu qui servait de secrétaire au pontife suprême des rnying-ma-pa m'ouvrit des possibilités inespérées de présence aux rituels et d'enregistrements en situation. Les années avaient passé : les camps précaires de réfugiés avaient fait place à des communautés structurées qui connaissaient certes encore bien des difficultés - le nombre des moines et moinillons ${ }^{4}$ s'était considérablement accru -, mais des centres d'enseignement avaient été mis en place et les moines étaient désormais habitués à la présence des Occidentaux, ils étaient familiarisés avec les appareils photo, les cameras, les radios, magnétophones et autres accessoires manipulés par les chercheurs et touristes; ils savaient que le bouddhisme se développait en Occident et que non seulement les fidèles occidentaux se multipliaient, mais qu'ils apportaient un soutien matériel indispensable à la survie et au développement de leurs propres communautés monastiques par des systèmes de parrainage. Même s'ils ignoraient pour la plupart les raisons de ma présence parmi eux, cette présence ne les surprenait plus, j'étais supposée avoir l'accord de l'abbé ou du maitre de chant et présumée apporter une manne financière bienvenue ou même assurer un parrainage qui me fut demandé à maintes reprises.

\section{Les difficultés rencontrées/les obstacles à vaincre}

Y eut-il des difficultés spécifiques dûes à ma situation de femme travaillant dans une communauté d'hommes? On peut se poser la question, et du reste mes collègues masculins n'avaient pas manqué d'attirer mon attention sur ce point! Mais je ne le crois pas et, de toutes façons, lorsque j'ai commencé à m'intéresser aux manifestations sonores du bouddhisme tibétain, j'avais déjà largement dépassé la quarantaine et appartenais clairement à la catégorie des "mères» $(a-m a)$, dont on attend qu'elles se préoccupent sérieusement de la religion. Pourtant, le fait d'être femme avait un inconvénient, c'était d'empêcher que je puisse trouver asile dans l'enceinte du monastère, ce qui compliquait ma participation éventuelle à des rituels dont il était difficile de savoir d'avance le déroulement exact. Sur ce plan, la situation s'est nettement améliorée quand les monastères visités, soucieux d'accueillir visiteurs et pèlerins, ont prévu des locaux à proximité des lieux de culte, comme je l'ai expérimenté dans la communauté bonpo de Dolanji (en 1979) ou au monastère de Dpal-yul à Bylakuppe (en 1987) ; ultérieurement plusieurs monastères ont ouvert des «Guest Houses » dont j'ai pu bénéficier, comme ce fut le cas à Bodnath, depuis 1991. Il n'était pas difficile alors de passer et repasser au monastère pour s'informer, surveiller les opérations en cours, obéir à l'appel du gong qui 
sonnait pour convoquer les moines selon des horaires dont la régularité était loin d'être assurée.

Je n'insisterai pas sur les difficultés que j'appellerai d'ordre politico-administratif : elles auraient été les mêmes quel que soit le domaine de recherche auquel je me serais attaquée, mais je signalerai néanmoins ma déception quand, à peine arrivée au Ladakh, je découvris qu'il ne m'était pas permis de séjourner à proximité du monastère de Phyang qui était situé dans une zone géographique jugée trop proche de la frontière chinoise et se trouvait être le seul monastère dont je possédais les notations musicales, objet de mon étude ! La visite au monastère fut extrêmement brève, puisque effectuée sans permis : elle me permit tout juste d'effectuer deux heures d'enregistrement avec un groupe de dix moines (Helffer, 1978) et je dus inviter le maître de chant à se déplacer à la capitale Leh pour pouvoir lui poser quelques questions et discuter avec lui du contenu d'un texte explicatif inclus dans le manuel de notations.

31 Ailleurs, on a parfois opposé à ma demande une interdiction administrative de séjourner dans les camps de réfugiés tibétains, ce qui m'a empêchée d'entreprendre un véritable travail à Tashijong au sein de la communauté établie autour du huitième Khams-sprul Rinpoche Don- rgyud nyi-ma (1930-1986); il faut dire que, dans ce cas précis, j'avais d'autres obstacles à vaincre: un chercheur duquel je me recommandais n'avait pas respecté ses engagements et avait omis ou oublié d'envoyer les copies d'enregistrements qu'il avait promises ; un autre avait déjà enregistré plusieurs rituels en vue de publier des disques et avait sans doute fait miroiter les avantages matériels de son entreprise et, pour ma part, je n'avais que l'intérêt scientifique à mettre en avant !

Enfin, à deux reprises, au cours de ma première mission de 1973, il me fut absolument interdit d'assister aux rituels qui se déroulaient à l'intérieur du monastère: on m'expliqua que ces rituels ne concernaient que les moines qui avaient reçu les initiations correspondantes et que, en conséquence, les laïcs tibétains ou étrangers ne pouvaient en aucun cas y être admis.

Mais le handicap principal que j'eus à surmonter résultait de mes lacunes personnelles : une insuffisante maîtrise de la langue tibétaine parlée aussi bien qu'écrite, une connaissance trop superficielle $\mathrm{du}$ bouddhisme en général, de la place et des particularités des différentes écoles, des liens ou des rivalités entre monastères, des rapports hiérarchiques existant entre membres d'une même confession, l'ignorance où, au temps de mes premières missions, je me trouvais du vocabulaire technique concernant la musique et la pratique du chant rituel, etc. Ceci m'a sûrement valu un certain nombre de gaffes : j'ai ainsi parfaitement conscience que, accueillie au monastère rnying-ma-pa de Bylakuppe, fondé par Padnor Rinpoche, je n'ai pas honoré comme je l'aurais dû le chef spirituel de la communauté, car je m'étais située dans la mouvance d'un autre maitre spirituel, Dilgo Khyentse Rinpoche (1910-1991), à l'époque pontife reconnu des rnyingma-pa, qui dispensait des enseignements dans le monastère. C'est pourtant bien Padnor rinpoche qui, entre autres publications, avait édité en 1984 les deux manuels de notations musicales du monastère de Dpal-yul au Khams, c'est lui qui avait fait venir de ce Dpal-yul tibétain (par opposition au Nouveau Dpal-yul reconstitué en Inde) un dbu-mdzad compétent qui animait toutes les célébrations, c'est lui qui avait désigné les jeunes joueurs de hautbois rgya-gling les plus compétents pour effectuer les enregistrements qui m'intéressaient et c'est encore lui qui, lorsque je vins lui présenter ma modeste offrande au moment de mon départ, m'indiqua des références précieuses de textes concernant 
l'origine du tambour rnga, c'est enfin lui qui, depuis la disparition de Dilgo Khyentse Rinpoche, est reconnu comme le chef de file des rnying-ma-pa.

La complexité des rituels constituait et continue à constituer une des difficultés majeures à vaincre : on a beau se débrouiller passablement pour lire les textes (qui peuvent utiliser des écritures différentes), il faut en effet jongler entre des textes différents dont seuls quelques passages seront utilisés. En outre, durant les longs rituels dont le déroulement s'étale sur plusieurs jours, si la structure générale du rituel celébré quotidiennement demeure la même, certaines parties sont différemment solennisées selon les jours : il en résulte que, pour entendre la totalité des chants de type dbyangs correspondant à un rituel donné, il faut assister tous les jours à la totalité du rituel, à raison d'une dizaine d'heures de présence par jour, ce qui entraîne une fatigue certaine pour les moines aussi bien que pour le chercheur de service. C'est pourtant le seul moyen d'observer les conditions exactes de l'emploi des divers instruments de musique et de choisir les passages qui pourront ou devront faire l'objet d'un enregistrement. Et surtout, c'est seulement sur place, en interrogeant les praticiens du rituel, qu'on peut apprendre les règles qui président au choix des différentes séquences et donc les conséquences qui en résultent au plan musical.

\section{Quelle attitude ai-je adoptée?}

Je ne saurais parler ici d'une réelle méthode de travail ; ma démarche, on l'aura compris, est demeurée empirique; néanmoins un certain nombre d'attitudes se sont imposées à moi et elles se sont modifiées au cours des années.

Si je me reporte à mes premières missions, j'étais en visite et j'étais demanderesse, je venais pour obtenir quelque chose en vue du double objectif que je m'étais fixé : la collecte des manuels de notations musicales utilisés dans les diverses traditions du bouddhisme tibétain d'une part, la réalisation d'enregistrements illustrant ces manuels d'autre part ; je demeurais extérieure à la vie du monastère dont j'ignorais les règles de fonctionnement, je profitais avec plus ou moins de succès des occasions qui se présentaient, j'accumulais de la documentation que je m'efforçais péniblement de décrypter à mon retour.

Par la suite, au fur et à mesure que je prenais davantage conscience de la place tenue par les chants vocalisés de type dbyangs - ceux qui précisément faisaient l'objet d'une notation musicale - dans la continuité des rituels, la nécessité d'enregistrer des rituels en situation se fit davantage sentir. A force de lire et relire les divers manuels de notation dont je disposais, j'avais plus ou moins compris (ou cru comprendre ?) l'enseignement qu'ils avaient fixé, je m'étais familiarisée avec la terminologie concernant le chant, l'emploi des cymbales et des tambours, le jeu des trompes et des hautbois ; parallèlement, j'avais découvert l'existence de textes tibétains relatifs aux instruments de musique qu'il me fallait traduire, ce qui me renvoyait inéluctablement à un travail livresque que je ne pouvais poursuivre qu'avec l'aide des tibétains eux-mêmes, sur le terrain ou à Paris. Oserais-je dire que je commençais à envisager la musique rituelle du bouddhisme tibétain un peu comme les Tibétains eux-mêmes, c'est-à-dire non pas comme une discipline artistique à cultiver pour elle-même, mais comme la part sonore du rituel, dont l'exécution correcte était indispensable à l'efficacité de ce rituel ? 
Petit à petit, avec une meilleure connaissance des rituels, j'avais le sentiment de devoir passer de mes visites en quelque sorte touristiques à une immersion dans le rituel luimême et je me demande si cette évolution ne s'apparente pas, au moins partiellement, à celle des jeunes moines qui assistent d'abord au rituel sans en connaitre le sens ou en comprendre le déroulement et deviennent progressivement capables de suivre le texte et éventuellement de se joindre aux différentes formes de psalmodie, en commençant par les récitations syllabiques, puis en s'initiant, s'ils en sont capables et en ont le goût, au chant proprement dit. Bien sûr, il n'était pas question pour moi de me mêler au chant: que serait venue faire une voix féminine au sein de ce choeur d'hommes? Je m'installais derrière les plus jeunes moines, pas trop loin des musiciens joueurs de trompes et de hautbois pour suivre leurs interventions, à une place où je pouvais voir l'attitude du " maître de chant ", et je suivais avec zèle sur les manuels, devenant capable d'apprécier la qualité d'une exécution ou de répérer les fautes éventuelles par rapport au manuel de chant! Puis, au bout de trois ou quatre jours, lorsque l'exécution du rituel avait pris en quelque sorte sa vitesse de croisière, et que j'en avais bien distingué les enchaînements ${ }^{5}$, je plaçais discrètement mes micros de façon à ne pas interférer dans la liturgie qui comporte de nombreux déplacements et j'enregistrais les passages qui me semblaient significatifs. Ce n'est qu'après cette immersion dans la durée des rituels que je devenais capable d'en apprécier la dramaturgie, et que, sensible aux oppositions de timbres, aux modifications de tempo, à la tension extrême de certains passages, je cessais de me comporter en simple spectateur. Je n'étais pas devenue bouddhiste pour autant, quoique je me sois parfois posé la question de l'opportunité de «prendre refuge dans le Bouddha, la loi (bouddhique), et la communauté bouddhique » selon la formule consacrée et que j'aie finalement écarté cette hypothèse, tant par conviction religieuse personnelle que par respect pour les convictions de la communauté bouddhique qui m'accueillait. Manifestant le plus grand respect pour la démarche des fidèles bouddhistes, il ne me paraissait pas honnête de feindre une appartenance qui n'était pas la mienne, même si cette démarche devait faciliter mon travail d'ethnomusicologue. Dans ces conditions, il ne pouvait donc pas s'agir d'observation participante, en tout cas pas dans le sens où on l'entend habituellement. 
Fig. 2 : Procession de jeunes moines lors d'une cérémonie au monastère de Zhe-chen, Bodhnath, Népal, 1991.

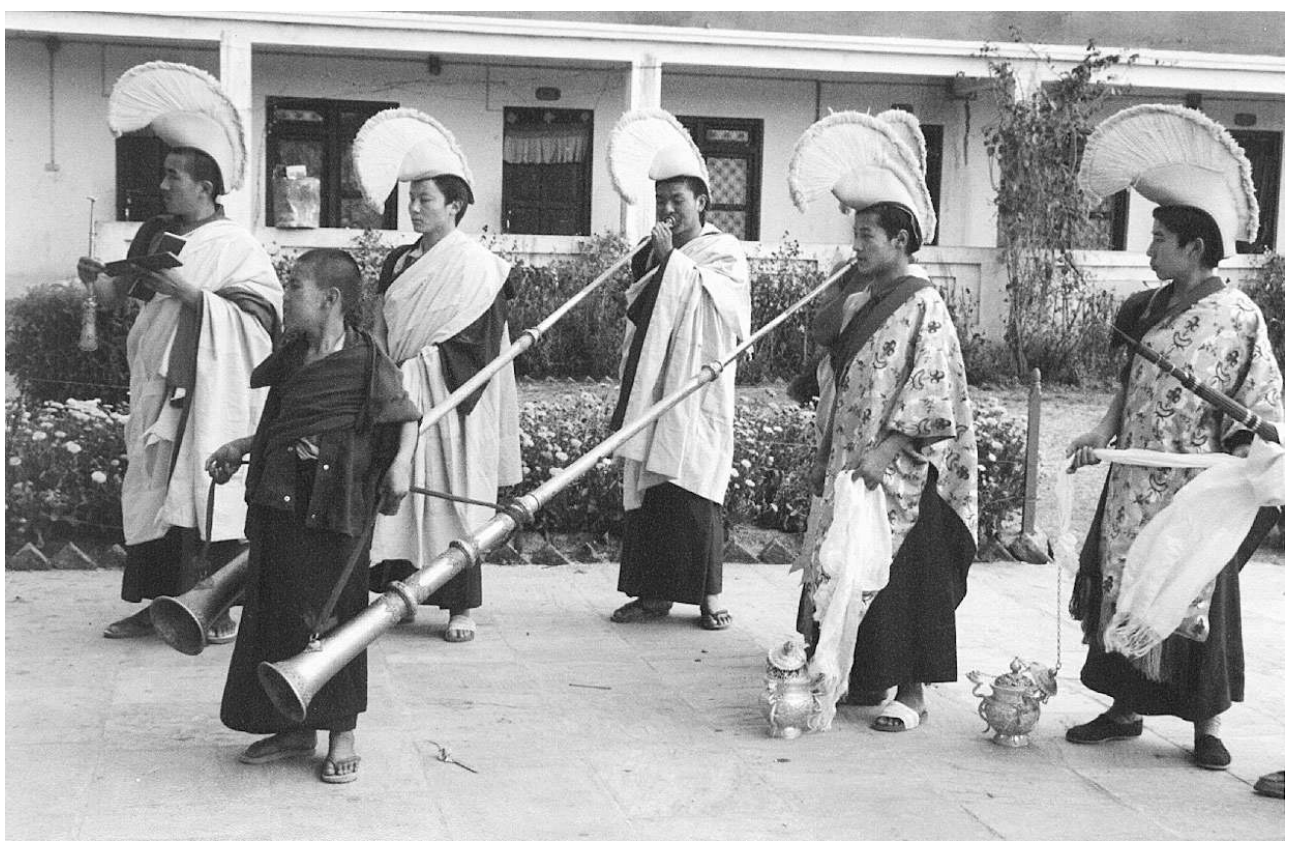

Photo : Mireille Helffer.

textes et d'enregistrements, j'eus à répondre à une demande émanant de la communauté monastique, sous forme d'un échange de services: puisque je disposais d'un matériel d'enregistrement, il me fut demandé de bien vouloir enregistrer, hors situation mais avec le concours d'un maître de chant confirmé, le contenu du manuel de chant de la tradition rnying-ma-pa de Mchog-gyur gling-pa (1829-1870), pour transmettre cet enregistrement à une communauté bouddhique implantée en Dordogne et désireuse de suivre ce modèle liturgique. Le $d b u-m d z a d$ qui se prêta à l'entreprise était celui-là même qui, réfugié en Inde, avait reconstitué de mémoire le contenu du manuel de chant et il comprenait fort bien le complément indispensable que constituait un support sonore pour transmettre la tradition dont il était le seul dépositaire en Inde.

C'est pour répondre au même type de requête que, en 1987, lors de mon séjour au monastère rnying-ma-pa de Dpal-yul à Bylakuppe, j'enregistrai avec l'excellent dbu-mdzad du monastère, le contenu d'un manuel, à l'intention cette fois d'un autre monastère rnying-ma-pa, celui de Zhe-chen dont l'installation à Bodnath au Népal était récente et qui, sur les instances de Dilgo Khyentse Rinpoche, ajoutait de nouveaux rituels à son répertoire.

41

fin, tout récemment (en mars 1995), à ma cinquième visite au monastère de Zhe-chen, Rabjam Rinpoche, à la fois supérieur et principal «réincarné » du monastère m'a fait savoir qu'il souhaitait conserver trace dans les archives du monastère des exécutions fournies par le dbu-mdzad Nor-bzang, dont la voix et la formation au Tibet fournissaient une référence particulièrement valable pour la transmission du répertoire de Zhe-chen. Après plusieurs visites à Zhe-chen, j'avais pu moi-même apprécier les compétences exceptionnelles de ce maître de chant, par rapport à ceux qui étaient en exercice les années précédentes. C'est donc avec plaisir et reconnaissance que j'ai enregistré 
systématiquement le contenu de deux manuels de chant correspondant au rituel de gtorzlog ou dgu-gtor ${ }^{6}$ auquel je venais d'assister du $22^{\mathrm{e}}$ au $29^{\mathrm{e}}$ jour du douzième mois de l'année tibétaine (22 fév.- 28 fév. 1995), juste avant la fête du Nouvel An tibétain.

Le responsable du monastère avait décidé, son secrétaire et assistant, le moine français Matthieu avait organisé, je n'eus plus qu'à fonctionner comme preneur de son (avec comme supériorité sur un simple technicien que je pouvais suivre exactement le manuel de référence et prévoir les enchaînements): en cinq séances d'enregistrements réalisés dans la bibliothèque du monastère, à l'écart des agitations coutumières, une quinzaine d'heures ont ainsi été enregistrées, alors que je pouvais suivre sur la "partition» tibétaine l'exécution du soliste qui, conscient de sa responsabilité, suivait scrupuleusement les indications du texte.

43 Je ne pense pas qu'une telle opération eut été envisageable quelques années auparavant ; disposer de tels documents était inespéré, mais en même temps posait en des termes nouveaux pour le chercheur que j'étais le rapport de l'écrit et de l'oral. Il était incontestable que, dans ce domaine, comme dans les autres domaines de la tradition tibétaine, le document écrit, en l'occurence le texte du rituel et la notation qui le complétait, accompagnés éventuellement du «mode d'emploi » (lag-len), constituaient la référence - le modèle ou "l'émique ", selon le vocabulaire en usage parmi les ethnomusicologues -, mais simultanément, il n'était pas question d'avoir accès à ces références par soi-même, il fallait en recevoir la «transmission » par un « maître » - nul rituel ne débute sans la récitation de la lignée de transmission (brgyud-'debs) de ce rituel -. La transmission des traditions proprement musicales était l'affaire de spécialistes dont la place dans la hiérarchie monastique était bien définie, elle relevait de la compétence des "maitres de chant» qui l'avaient eux-mêmes reçue de leurs prédécesseurs dans le monastère et devaient la conserver précieusement, d'où le souci permanent des responsables des monastères de l'exil de retrouver parmi les rescapés du drame tibétain des maîtres de chant fiables.

Dans ces conditions, on peut se demander quel est le document sonore qui revêt la plus grande importance ? Est-ce l'enregistrement fait « en situation ", pendant le rituel, alors que les moines participants (et les jeunes moines chahuteurs!) sont souvent peu capables - et c'est un euphémisme - de suivre le modèle énoncé par le dbu-mdzad et que les différentes actions liturgiques à effectuer, les déplacements des uns et des autres, la récitation d'un mantra qui ne doit s'interrompre à aucun moment pour toute la durée du rituel, concourent à brouiller le résultat sonore... ce qui rend une exploitation « scientifique » difficile ? Est-ce le document enregistré par le seul "maître de chant », hors situation? Faut-il enregistrer avec ou sans accompagnement des cymbales et/ou du tambour qui sont toujours joués par le « maître de chant » responsable de la coordination musicale, mais dont le son puissant et la résonance prolongée nuisent souvent à la clarté du texte énoncé?

Je dois reconnaître que j'ai la plupart du temps été guidée par les circonstances qui ont rarement été idéales et se sont toujours écartées de la situation dans laquelle ont été effectués de nombreux enregistrements commerciaux largement diffusés mondialement, enregistrements effectués en studio, ou lors des «tournées » effectuées par les moines d'un monastère pour recueillir les fonds destinés à quelque nouvelle construction ou à l'entretien des moines. J'ai donc, dans la mesure du possible, privilégié l'enregistrement en situation pendant le rituel, même si c'était au détriment de la qualité sonore obtenue. 

chant» -, je demeure perplexe sur le rôle que peut jouer l'ethnomusicologue dans la conservation des dites traditions : si la collecte et la conservation des documents dans des archives appropriées me paraît indispensable, c'est aux tibétains et aux responsables religieux qu'il revient de choisir l'usage qu'ils en feront et je me contenterai de leur avoir facilité la tâche à un moment difficile.

C'est donc à une extrême modestie que me conduit la fréquentation des monastères : je leur suis pleinement redevable à tous les plans, accueil, communication des données écrites, autorisation d'assister aux rituels, contacts avec les maîtres de chant. Que puis-je leur donner en échange? Certes je peux contribuer à fixer par l'enregistrement des traditions évanescentes comme celles du chant ou d'une voix particulière, mais force m'est de convenir que toutes les notations auxquelles je m'efforcerai de parvenir, toutes les analyses au sonagraphe, si utiles soient-elles pour élucider avec plus de rigueur les caractéristiques d'une tradition, satisferont peut-être la curiosité insatiable des savants musicologues d'Occident, mais ne sont d'aucune utilité pour mes informateurs.

Force est de constater que, en 1995, la situation des Tibétains a évolué : les monastères en territoire occupé ont parfois retrouvé une part de leurs activités, mais ils manquent de maîtres compétents et leur situation matérielle est plus que précaire : dans de nombreux cas, ils doivent compter sur l'aide des Tibétains exilés; les monastères de l'exil, soutenus par les fidèles bouddhistes d'Occident, de Taiwan, de Malaisie, se sont multipliés et recrutent abondamment; l'action persévérante du quatorzième Dalaï lama, Prix Nobel de la Paix, a fait connaître à toute la planète les positions du bouddhisme tibétain et les media s'emparent fréquemment d'un sujet aussi porteur ; les communautés bouddhiques se développent en Occident, attirant un nombre grandissant de fidèles zélés, mais les dangers de voir s'affadir et disparaître une tradition musicale à la fois unique et diversifiée demeurent bien réels.

Les moines tibétains de la jeune génération, ceux qui sont nés en exil ou hors du terreau proprement tibétain, auront-ils à coeur et pourront-ils maintenir des traditions liturgiques matériellement aussi lourdes ? Ne vont-ils pas devenir (s'ils ne l'étaient déjà ?) de simples fonctionnaires religieux à la situation sociale enviable? Ne va-t-on pas assister à une simplification progressive et à une uniformisation des rituels dont le maintien n'est plus jugé nécessaire par une communauté tibétaine absorbée par la course à l'argent? L'Occident pourra-t-il maintenir l'effort consenti à l'égard des monastères dont l'entretien coûte cher? Assistera-t-on à un transfert des traditions du bouddhisme tibétain vers l'Ouest, avec toutes les adaptations que cela peut impliquer?

Quoi qu'il en soit, étant donné le processus engagé, la conservation des traditions musicales relatives aux liturgies du bouddhisme tibétain demeure menacée, la continuation des opérations destinées à recueillir des enregistrements des principaux rituels pendant qu'il en est encore temps reste une des missions prioritaires à remplir dans les années qui viennent, en même temps qu'une analyse de leur contenu permettra d'en mieux comprendre la structure et les caractéristiques musicales. Il serait souhaitable que, comme on l'a vu au Japon dans la première moitié $d u x^{e}$ siècle, les autorités religieuses tibétaines, alertées par les chercheurs occidentaux qui se mettraient à leur service, en prennent davantage conscience et aient à coeur de constituer les archives sonores qui peuvent leur devenir précieuses dans un proche avenir. Les missions de terrain initiées depuis une vingtaine d'années par ethnologues, musiciens ou 
ethnomusicologues auraient montré la voie à suivre et n'auraient pas alors été effectuées au seul bénéfice de la science occidentale.

\section{BIBLIOGRAPHIE}

CANZIO Riccardo, 1978, Sakya Pandita's Treatise on Music and its Relevance to Present Day Liturgy, unpublished Ph.D., London, School of Oriental and African Studies.

ELLINGSON Ter, 1979, The Mandala of Sound: Concepts and Sound Structures in Tibetan Ritual Music, unpublished Ph.D. in Philosophy, University of Wisconsin-Madison.

HELFFER Mireille, 1976, « Problèmes posés par la lecture des notations musicales (dbyangs-yig) utilisées pour le chant d'hymnes religieux au Tibet », In Etudes tibétaines, Actes du XXIXe Congrès des Orientalistes (Paris, juillet 1973), Paris, L'Asiathèque : 17-22.

HELFFER Mireille, 1978, Ladakh, musique de monastère et de village, 1 disque $30 \mathrm{~cm} / 33 \mathrm{t}$., Chant du Monde, collection CNRS/musée de l'Homme : Traditions musicales des cinq continents, LDX 74662. Réédition CD en 1989 sous LDX 274662, CM 251.

HELFFER Mireille, 1980, « The musical notation of the hymn rTsa-brgyud-ma in the dGe-lugs-pa tradition ", In Tibetan Studies in honour of Hugh Richardson, Proceedings of the International Seminar of Tibetan Studies Oxford 1979, M. Aris and Aung San Suu Kyi ed., Warminster, Aris and Philips Ltd : 120-131.

HELFFER Mireille, 1990, « Recherches récentes concernant l'emploi des notations musicales dans la tradition tibétaine », In Tibet, Civilisation et Société, Paris, Fondation Singer-Polignac : 59-84.

HELFFER Mireille, 1993, « Tibetan Monasteries in the Valley of Kathmandu and their Role in the Preservation of Musical Traditions : the Case of the Monastery of Zhe-chen ", In Nepal, Past and Present; Proceedings of the Franco-German Conference Arc-et-Senans, june 1990, G. Toffin ed., Paris, CNRS Editions : 205-214.

HELFFER Mireille, 1993, «A Recent Phenomena : the Emergence of Buddhist Monasteries around the Stupa of Bodnath ", In The Anthropology of Nepal : from Tradition to Modernity, Proceedings of the Franco-Nepalese Seminar held in French Cultural Centre, 18-20 mars 1992, G. Toffin ed., French Cultural Centre/ French Embassy : 114-131.

HELFFER Mireille, 1994, MCHOD ROL, Les instruments de la musique tibétaine, Paris, CNRS Editions/ Ed. de la Maison des Sciences de l'Homme [collection «Chemins de l'Ethnologie », dirigée par Gérard Toffin].

KAUFMANN Walter, 1975, Tibetan Buddhist Chant, Bloomington, Indiana University Press.

VANDOR Ivan, 1976, Bouddhisme tibétain, Paris, Buchet-Chastel [coll. « Les Traditions musicales », vol. VII]. 


\section{NOTES}

1. A l'exception de la tentative faite par le Pr. Graf à partir des enregistrements réalisés par le tibétologue R. de Nébesky-Wojkowitz (Graf, 1962), entreprise qui souffrait de graves erreurs méthodologiques dues à une approche trop ethnocentrique et à l'ignorance de la langue tibétaine.

2. cf. Helffer 1976

3. Jusqu'à l'invasion chinoise, c'est traditionnellement au monastère de 'Bri-gung, fondé au XII siècle au Tibet central, que les moines du monastère de Sgang-sngon à Phyang, allaient faire leurs études ; ils peuvent donc être considérés comme dépositaires de la tradition de 'Bri-gung.

4. Nombre de familles tibétaines confient au monastère leurs enfants âgés souvent de 6 ou 7 ans.

5. Le rituel se déroulait habituellement en deux grandes sessions à peine interrompues par les distributions de thé au beurre (à peu près toutes les heures) : la session du matin commençait aux environs de $6 \mathrm{~h} 30$ et durait jusqu'à l'heure du déjeuner vers $11 \mathrm{~h} 30$; l'après-midi les opérations reprenaient vers $13 \mathrm{~h} 00$ pour s'arrêter vers $18 \mathrm{~h} 00$. Lorsque des cérémonies supplémentaires devaient être ajoutées, comme c'était le cas pour les ballets rituels des $28^{\mathrm{e}}$ et $29^{\mathrm{e}}$ jours, le rituel commençait plus tôt et la totalité des offices exécutés les jours précédents prenait place dans la matinée.

6. La célébration de cet important rituel, destiné à évacuer tous les maux de l'année écoulée, requiert la fabrication de gâteaux rituels (gtor-ma), parmi lesquels figurent une représentation de Gshin-rje-gshed - celui qui est vainqueur de la mort - et de son assistant Las-gshin. Le $29^{\mathrm{e}}$ jour, les gtor-ma sont solennellement détruits - d'où le nom par lequel on désigne le rituel : « les gtorma du $29^{\mathrm{e}}$ jour (dgu-gtor) ».

\section{RÉSUMÉS}

La fréquentation régulière de nombreux monastères tibétains, en Inde (Ladakh, Himachal Pradesh, Karnataka) et au Népal, permet de procéder à une évaluation de l'évolution d'une pratique de terrain qui s'étend sur plus de deux décennies. Après une période de collecte « extensive » de matériaux écrits (notations musicales, textes de rituels) et sonores (des dizaines d'heures d'enregistrements) permettant une approche comparative du domaine, s'est fait sentir le besoin d'une connaissance plus approfondie d'une tradition particulière. C'est ainsi que, petit à petit, s'est précisé un attachement à un monastère de la tradition rnying-ma-pa, celui de Zhechen à Bodnath (Népal), où l'auteur séjourne dorénavant plusieurs semaines par an au moment de la célébration des grands rituels de fin d'année. A la simple collecte de matériaux au cours de séjours d'une durée limitée, s'est substituée une écoute attentive et une véritable immersion dans des rituels complexes dont il devient possible de saisir les articulations et les particularités musicales ; la familiarité accrue avec les moines, le maître de chant, les autorités du monastère, permettent des échanges fructueux dont il est donné quelques exemples.

Frequent visits to numerous Tibetan monasteries, both in India (Ladakh, Himachal Pradesh, Karnataka) and in Nepal, allow one to carry out a study of fieldwork practices covering two decades. After a period of 'across the board' collection involving written material (musical scores 
and ritual writings) and sound (ten or so hours of recording); enabling a comparative study of the domain, the need was felt for a more in depth study of a particular aspect. Thus in this way, a closer involvement with a monastery of the rnying-ma-pa tradition, Zhe-chen in Bodnath, Nepal came about. Here, the author stayed several weeks a year when the end of the year ritual celebrations took place. Straightforward collection of material during short stays was replaced by attentive listening and a genuine involvement in complex rituals of which it became possible to grasp the structure and distinctive musical features; increased informality with the monks, the song master and the monastic authorities allowed for fruitful exchanges, some examples of which are cited here.

\section{AUTEUR}

\section{MIREILLE HELFFER}

Mireille Helffer, née le 6 janvier 1928, directeur de recherche honoraire au CNRS, chargée de mission au Musée national des Arts Asiatiques-Guimet, membre de l'UMR 9957 (Laboratoire d'ethnomusicologie) qu'elle a dirigée de 1986 à 1990, a contribué à la mise en place de l'enseignement de l'ethnomusicologie à l'Université de Paris X-Nanterre où elle a enseigné jusqu'en 1992. Spécialiste des musiques himalayennes, elle a effectué de nombreuses missions de terrain au Népal, au Ladakh, et parmi les communautés tibétaines réfugiées en Inde. Ses publications ont touché à des domaines aussi divers que les castes de musiciens au Népal, le chant épique tibétain (objet de sa thèse de doctorat, publiée en 1977), le répertoire liturgique des monastères du bouddhisme tibétain, les notations musicales tibétaines, les instruments de la musique tibétaine. Elle se consacre aujourd'hui à l'étude des traditions musicales du monastère rnying-ma-pa de Zhe-chen au Népal. 